\title{
Responsabilidad social empresarial e innovación: Una mirada desde las tecnologías de la información y comunicación en organizaciones
}

\author{
Corporate social responsibility and innovation: a look from the information technology and \\ communication inorganizations
}

\begin{abstract}
Resumen: Partiendo de la revisión bibliográfica de las revistas de más alto impacto, se muestran los diferentes énfasis y tendencias teóricas de la discusión sobre el componente tecnológico en las organizaciones en los últimos seis años. Los aportes de los autores muestran la evolución de este concepto en su conjunto y su necesidad de incorporarlo para generar innovación tecnológica, mejoramiento en el desempeño organizacional y creación de valor.
\end{abstract}

El contenido y estructura del trabajo es resultado de la dinámica encontrada en torno a tres diferentes propuestas teóricas: Tecnologías de la Información y la Comunicación (TIC), la capacidad tecnológica y Responsabilidad Social Empresarial (RSE). La utilidad de este artículo es diversa. En primer lugar, se identificaron interrelaciones a partir de un análisis de las similitudes, diferencias y contraposiciones de los conceptos planteados entre los artículos revisados. Y en segundo lugar, sirve como evidencia de la necesidad apremiante de las organizaciones para que tecnológicamente se apropien de prácticas socialmente responsables que se traduzcan en una verdadera ventaja competitiva.

Palabras clave: Capacidad tecnológica, Desempeño organizacional, Impacto social, Responsabilidad Social Empresarial, Tecnologías de la Información y la Comunicación.

JEL: M10, M14, M15.

Abstract: Starting from the literature review of the highest impact journals, different emphasis and theoretical trends of the discussion on the technological component in organizations in the past six years they are shown. The contributions of the authors show the evolution of this concept as a whole and its need to incorporate it, to generate technological innovation, improvement in organizational performance and value creation.

The content and structure of the paper is the result of the dynamics encountered around three different theoretical proposals: information and communications technology (ICT), technological capability and Social Responsibility (CSR). The usefulness of this article is diverse. Firstly, interactions were identified from an analysis of the similarities, differences and contrapositions, of the concepts raised among reviewed articles. And secondly, it serves as evidence of the urgent need for

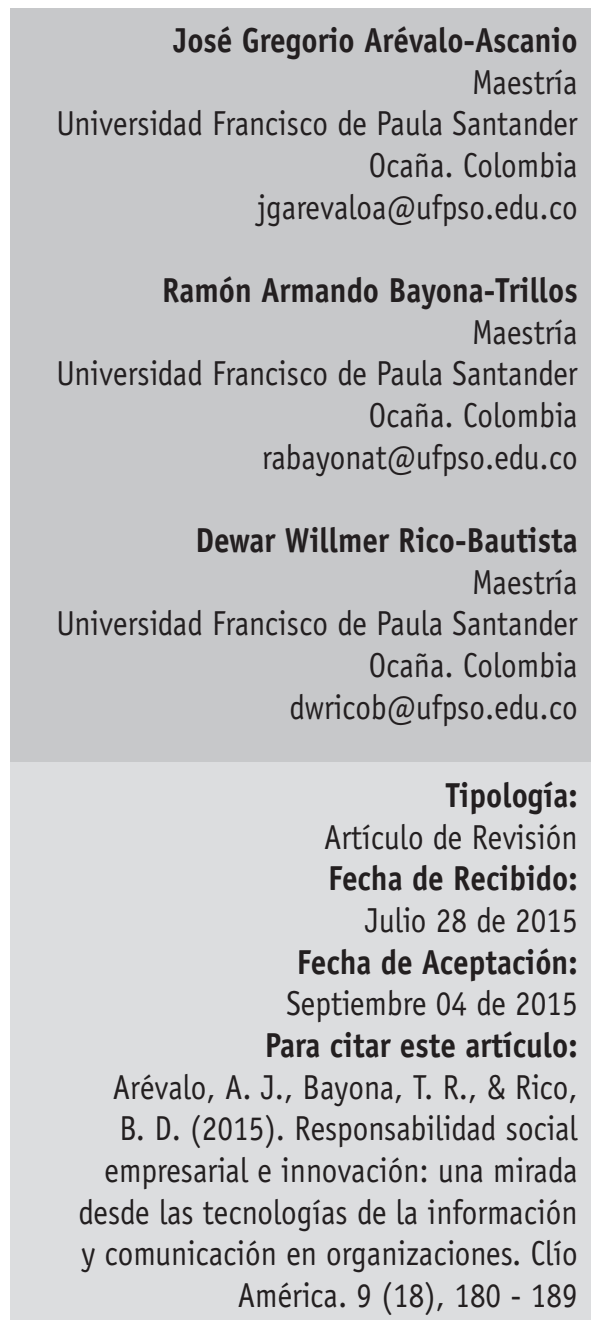


organizations to take ownership of technologically socially responsible

practices that translate into a true competitive advantage.

Keywords: Technological capacity, Organizational Performance, Social Impact, Corporate Social Responsibility, Information and Communications Technology

\section{Introducción}

El concepto de capacidad tecnológica se relaciona con elementos de gestión que guían el crecimiento y desarrollo sostenido y envuelve conocimientos, técnicas y habilidades para adquirir, usar, absorber, adaptar, mejorar y generar nuevas tecnologías (Lugones, Gutti, \& Le Clech, 2007); es decir, que incluyen las capacidades de innovación y las capacidades de absorción de tecnológica para su uso (García Muiña \& Navas Lopéz, 2007).

Por tanto es apremiante que las empresas a través de las TIC establezcan un vínculo más cercano con los actores involucrados en el entorno, cliente interno y cliente externo, permitiendo optimizar costos, estandarizar sistemas, tener información eficiente y oportuna para la toma de decisiones (Cano, 2013).

Este vínculo se complementa con lo descrito por Ibarra, Casas, y Olivas (2012) "el concepto de RSE debe ser incorporado a sus procesos de gestión administrativa y de calidad (...), preocupada por el concepto de sostenibilidad, en el que tienen cabida a un mismo nivel los rubros económico, social y medio ambiental" (pág. 3).

El proceso metodológico realizado se concibe desde una perspectiva sistémica, que pretende identificar similitudes y diferencias de los diversos planteamientos académicos, dando lugar a una síntesis de los variados aportes que se muestran alrededor de las áreas temáticas analizadas.

El uso de las TIC para lograr la competitividad en el sector productivo, ha motivado este trabajo de revisión de literatura especializada en el que se muestran los principales estudios en materia de acceso y utilización de las TIC, capacidad tecnológica y responsabilidad social empresarial en las organizaciones, además del gran impacto que tienen ellas para obtener una mayor productividad.

\section{Metodología}

El presente trabajo es una investigación teórico descriptiva de tipo documental, dado que el procedimiento implica la búsqueda, organización y análisis de un conjunto de documentos electrónicos (ver figura 1), sobre los temas de las TIC, capacidad tecnológica y responsabilidad social empresarial, entre los años de 2009 a 2014.

La revisión documental se realizó en revistas de alto impacto publicados en las bases de datos $A M J$, SCOPUS, SCIENCE DIRECT, SCiELO, Directory of Open Access Journals (DOAJ), The National Academies Press, REDALYC, y LATINDEX.

Como criterios de búsqueda, se incluyeron los siguientes descriptores: "Capacidad tecnológica", "Desempeño organizacional", "Impacto social", "Responsabilidad Social Empresarial", "Tecnologías de la Información y la Comunicación"; "Technological capacity", "Organizational Performance", "Social Impact", "Corporate Social Responsibility", "Information Technology and Communication". Estos descriptores fueron combinados de diversas formas al momento de la exploración con el objetivo de ampliar los criterios de búsqueda, en el período establecido.

Seguidamente, se explican las distintas partes que implicó la revisión documental: 


\section{Figura 1.}

\section{Proceso estructurado de revisión bibliográfica}

Identificación de

bibliografiarelevante.
Generación de áreas temáticas y tipologías
Interrelación entre artículos

Fuente: Elaboración propia.

\section{Identificación de bibliografía relevante}

El inicio del proceso consistió en realizar un rastreo documental en bases de datos especializadas, se preseleccionaron artículos y al final se seleccionaron cincuenta referencias, de acuerdo con los criterios de inclusión y exclusión. "No se tomaron en consideración para el análisis aquellos artículos que no hacían alusión a los núcleos temáticos y/o aquellos que no se encontraban en revistas indexadas" (Sánchez , 2011, p. 179).

\section{Generación de áreas temáticas y tipologías}

Como acto seguido, se analiza cada documento, se establecen sus áreas temáticas y tipologías, como punto de entrada del proceso de revisión. Partiendo de un formato elaborado en word con los siguientes campos: título del artículo, autor, año, revista, información de la revista, problema de investigación, objetivos, tipo de investigación, método, descripción, instrumentos utilizados, resultados y núcleo temático. En este aparte, se pretendió identificar puntos de encuentro y diferencia de criterios entre los documentos clasificados que fundamentan la estructura de los ejes descritos para el presente artículo.

\section{Interrelación entre artículos}

En este aparte, se pretendió identificar puntos de encuentro y diferencia de criterios entre los documentos clasificados que fundamentan la estructura de los ejes descritos para el presente artículo. Este proceso derivó en un extenso, que tuvo que ser resumido para cumplir con los lineamientos de publicación de artículos. Finalmente, se realizó un análisis global mediante el cual se identificaron las convergencias y divergencias del análisis de cada uno de los núcleos temáticos, se formularon ciertas hipótesis y conclusiones. (Sánchez , 2011, p. 180)

\section{TIC en la dinámica organizacional}

Los estudios de los últimos 10 años sugieren que las TIC pueden ofrecer amplias oportunidades al sector productivo, entre ellas, lograr una mayor cobertura en el mercado y por ende un mejor posicionamiento y la mejora de las relaciones con los distintos grupos de interés. No hay duda de que los cambios presentados en estos años han generado una transformación productiva que no puede entenderse sin tener en cuenta las TIC. 
Lo anterior, coincide con Venteño, De la Rosa, Casas, Trujillo, y Fuentes (2010) quienes señalan que: "la mejora en el desempeño a través de las tic, una vez atravesada la etapa de informatización de los procesos de apoyo (información) y de articulación de las diversas áreas (comunicación), depende del grado de avance en la introducción de herramientas TIC" (p. 9).

Por tanto, el uso de las TIC para lograr la competitividad en el sector productivo, ha motivado este trabajo de revisión de literatura especializada en el que se muestran los principales estudios en materia de acceso y utilización de las TIC por parte de las empresas, además del gran impacto que tienen ellas para obtener una mayor productividad.

De la misma manera, este acápite pretende crear conciencia acerca de la posición que deben tomar las empresas en la era de las TIC para asegurar una adecuada consecución de los objetivos organizacionales.

Estas son algunas de las investigaciones que se han realizado en torno a las Tecnologías de la Información y la Comunicación (TIC) y su aplicación en las empresas:

Correa, Gómez, y Cano (2010), a través de su investigación titulada Gestión de almacenes y tecnologías de la información y comunicación, se identificó por una parte, que las tecnologías aplicadas a la gestión de almacenes contribuye a la simplificación de las operaciones, reducción de costos y mejoras de los flujos de información y por otra que los principales problemas para la implementación de las mismas son los altos costos y la cultura organizacional.

Uno de los campos en los que las TIC también ha efectuado su trabajo es el universitario, específicamente en la gestión gerencial de los docentes en una universidad, Poriet y Martínez (2014) realizaron un estudio en el que identificaron las competencias básicas en Tecnologías de Información y Comunicación (TIC) que posee el docente en el ejercicio de funciones gerenciales en una universidad venezolana.

Por otra parte, se obtuvo información de casos mexicanos a partir de los trabajos realizados en primer lugar por Venteño et al. (2010), en el que se aborda el avance en las Tecnologías de la Información y Comunicación (TIC), desde la perspectiva de las empresas y particularmente la actividad bancaria tomando como punto de partida el contenido del Manual de Lisboa, específicamente el capítulo denominado "Acceso y uso de las tic aplicado a la empresa" y en segundo lugar por Saavedra y Tapia (2013), quienes entrevistaron a 394 empresas del sector industrial y así determinar el dominio sobre su uso de las TIC, encontrando que las Mipyme industriales en México tienen bajas probabilidades de sobrevivencia en el largo plazo.

En este mismo país, se encontró la investigación desarrollada por González y Casalet (2004), en donde plantean una reflexión sobre el uso de las Tecnologías de la Información y Comunicación (TIC) en las pequeñas y medianas empresas mexicanas (Pymes), en términos de los cambios en los procesos de producción y organización, en el entorno institucional público-privado y las ventajas de esto en la creación de capital social.

De forma general, a nivel latinoamericano, es pertinente hacer alusión al trabajo realizado por Scheel Mayenberger y Rivera Gonzales (2009), cuyo objetivo fue demostrar cómo son utilizadas las TIC dentro de las empresas latinoamericanas con el fin de analizar su situación actual y al mismo tiempo proponer posibles mejoras. En este mismo trabajo se enfatiza en la necesidad de rediseñar a la empresa latinoamericana incorporándola a ciclos apalancados por las tecnologías de información y telecomunicaciones.

Dentro de los estudios recientes aparece Alderete, Jones, y Morero, (2014), como un trabajo que hace un aporte a la literatura sobre los determinantes de la adopción de las nuevas Tecnologías de la Información y de la Comunicación (TIC) a nivel empresarial, en el sector industrial de Argentina, específicamente en los sectores automotriz y siderúrgico. Se pudo identificar que los factores que influyen positivamente en la adopción de las TIC en estas industrias son: la pertenencia a un grupo económico, el nivel global de innovación y el nivel de aprendizaje interno de la empresa.

A lo largo de la literatura especializada revisada sobre los trabajos que estudian la incidencia de 
las TIC en el desempeño organizacional, se pudo encontrar el estudio desarrollado por Gargallo y Pérez (2009), cuyo objetivo principal fue el mostrar las características diferenciadoras de las empresas de Economía Social frente al resto, en cuanto a su eficiencia y al uso de este tipo de tecnologías, para esto se tomó una muestra representativa de las empresas manufactureras españolas.

A nivel de empresa, los estudios han sido muy diversos, así lo demuestra el trabajo realizado por Sandulli, Fernández, López, y Rodríguez (2007), con el que se analiza la eficiencia técnica de las firmas españolas utilizando el modelo Data Envelopment Analysis (DEA) y se muestra en sus hallazgos que no es suficiente invertir en TIC para mejorar el desempeño organizacional sino que se requiere de una serie de inversiones adicionales y prácticas organizativas específicas.

Por su parte, otros autores que han abordado la temática, desde la perspectiva de los cambios en las estructuras organizacionales de las empresas son en primer lugar Vera, Labarcés, y Chacón (2011), donde su investigación fue dirigida a estudiar la evolución de las estructuras organizativas debido a la influencia de las TIC y la forma en que estas han propiciado la aparición de nuevas formas de organización como las llamadas organizaciones virtuales, de redes y celulares. En este mismo sentido, aparecen Berné, García, Esperanza, y Múgica, (2011), quienes con su investigación pretendieron analizar y diagnosticar un posible cambio estructural de la distribución del sector turístico en España, afectada por el uso intensivo de las TIC, revelando un uso predominante de los medios electrónicos en los canales de distribución turísticos y mostrando un reajuste actual en el sistema, que afecta a las posiciones de los intermediarios y confirma al consumidor como un elemento clave.

Para el caso de Colombia, la revisión de los estudios muestra tres investigaciones halladas; por un lado, la desarrollada por Alderete M. (2012), con ella se aporta a la medición del avance de las nuevas TIC a nivel empresarial en el sector de servicios de Colombia. Su objetivo consistió en construir un índi- ce sintético o compuesto, a partir de tres subíndices compuestos alternativos tales como: uso de las TIC, preparación para el uso de las TIC e impacto; en segundo lugar, lo realizado por Salazar, Vanegas, y Arboleda, (2009), quienes adelantaron un estudio de casos realizado en 10 empresas del Valle de Aburrá, Antioquia, que han incorporado tecnologías digitales a la gestión de comunicación interna, en ésta se encontró que el uso y la aplicación de las tecnologías que están incorporando los comunicadores organizacionales a las herramientas digitales es de medios para el flujo informativo; y por último, la llevada a cabo por Montaño y Montoya (2010), la cual trata de establecer la utilización del comercio electrónico por parte de los empresarios medianos de Palmira, en ella se encontró que las empresas objeto de estudio, aunque cuentan con recursos de hardware y de conectividad, no poseen plataformas especializadas de comercio electrónico, razón por la cual sus ventas por internet son muy bajas.

La evidencia proporcionada por los resultados de las investigaciones analizadas en este eje temático es valiosa, ya que en ellos se examinaron los principales estudios en materia de acceso y utilización de las TIC por parte de las empresas y su incidencia como variable determinante para obtener una mayor productividad. Sin embargo, aunque hay estudios sobre esta temática con distintas fuentes y metodología, el avance ha sido menor en cuanto al nivel de incorporación de las TIC en las empresas.

Esto es un aporte clave, propio de la revisión, que muestra la evolución de las TIC en la dinámica organizacional, la preocupación ha variado desde la consideración de las mismas como un componente clave en la simplificación de las operaciones, reducción de costos y mejoras de los flujos de información. En términos organizacionales, esto quiere decir que con la incorporación de las mismas en los procesos empresariales, mostrada en la literatura da lugar a entender que las TIC en una organización deben ser consideradas con la finalidad de proveer herramientas para delinear una política institucional que permita apoyar la acumulación de capacidades tecnológicas, ya que son pocas las empresas que tienen una estrategia integral en este sentido. 


\section{La capacidad tecnológica: Un reto de innovación en las Organizaciones}

La capacidad tecnológica en los últimos años se ha convertido en uno de los elementos importantes en el desarrollo de las pequeñas economías y en especial aquellas donde son más sensibles a los cambios tecnológicos. Para Benavente (2005): "respecto de la innovación tecnológica se trata más de un proceso que de un resultado, (...) se necesita de insumos, los que se podrían agrupar en cuatro grandes categorías: capital financiero, capital humano, ideas e infraestructura" (p. 53). Lall (citado por Ezequiel, 2014) plantea la necesidad de que las empresas deben adquirir capacidades tecnológicas principalmente para mejorar la eficiencia. En este sentido, los tres factores que estimulan el desarrollo de las capacidades tecnológicas según el autor son: 1) necesidad interna del desarrollo de nuevas habilidades e información, donde esencialmente se busca optimizar la productividad; 2) factor externo, que influye fuertemente en el proceso; y 3) cambio tecnológico, que se desarrolla continuamente en casi todas las industrias del mundo desarrollado. Entender la dimensión y llegada de estos tres factores le permitió a Lall poder clasificar a las capacidades tecnológicas a nivel empresa por medio de la elaboración de una taxonomía que permite analizar la acumulación de las capacidades tecnológicas.

Desde una perspectiva global Albornoz (2001) afirma: "La importancia en la agenda internacional que se concede a las políticas para la ciencia, la tecnología y la innovación es creciente en los países industrializados" (p. 1). La experiencia de América Latina en utilizar la política científica y tecnológica como instrumento de desarrollo y competitividad, pese a ciertos logros en el plano académico, no puede ser considerada como un éxito. Algunos autores señalan que esto se debió a ciertos factores. El primero de ellos fue la escasa demanda de conocimiento científico y tecnológico por parte del sector productivo; el segundo factor tuvo carácter estructural y consistió en la inexistencia o la extrema fragilidad de los vínculos e influencias recíprocas entre el estado, la sociedad y la comunidad científica (Dagnino \& Hernán, 1999). En países como México existe el programa Especial de Ciencia y Tecnología (РECyT) 2001-2006, el cuál enfatiza en el papel de la innovación en las empresas vinculada con la competitividad (Treviño, 2002), donde plantean en uno de sus objetivos: "para elevar la competitividad y la innovación en las empresas se tiene que incrementar la inversión en actividades de investigación y desarrollo, (...), así como que el sector privado incremente su inversión en las actividades científicas y tecnológicas" (Parada , 2001, p.25), planteamientos que se relacionan con lo expuesto por Benavente citado en el artículo.

Estudios realizados en países como Argentina, Chile y Colombia demuestran la relación directa entre las capacidades de comercio exterior y las capacidades tecnológicas en las empresas; Milesi, Moori, Robert y Yoguel (2007) lo afirman: “El análisis de estas cuestiones se basa en datos de comercio exterior $y$ en información sobre los factores comerciales, (...), a partir de información obtenida de encuestas a algunas 300 pymes exportadoras (exitosas y no exitosas) de estos países" (p. 26). Los países en desarrollo y en particular los latinoamericanos han sido menos estudiados, la mayor parte de la información al respecto ha sido desarrollada con una visión evolutiva de la economía que enfatiza el papel de la tecnología y el conocimiento en el desarrollo de ventajas competitivas dinámicas (Milesi, et al. 2007).

Algunos hechos estilizados acerca de la incidencia de estos factores son: la teoría de la innovación señala que, además de las diferencias interindustriales derivadas de distintos perfiles de especialización productiva, también son muy importantes las diferencias tecnológicas intraindustriales (Dosi, 1988) (Nelson, 1991) (Freeman, 1994). Estos acervos, generalmente denominados capacidades tecnológicas, involucran aspectos tales como la calificación de los recursos humanos (habilidades), la forma que adopta la organización del trabajo y la existencia de estructuras formales e informales de investigación y desarrollo (Bell \& Pavitt, 1995) (Pietrobelli, 1996).

Particularmente en Colombia, en la década de los años noventa se vivieron importantes cambios en las condiciones para el avance de la CyT+I. La Ley 29 de 1991 que sentó las bases normativas para el desarrollo de estas actividades y la arquitectura institucional estructurada a partir de la constitución del Sistema Nacional de Ciencia y Tecnología; y en 
1995 la creación del Sistema Nacional de Innovación (SNI). Este avance planteó como reto el desplazamiento del énfasis en la CyT hacia la innovación y hacia la empresa como actor central del sistema (Pérez \& Rodríguez, 2004). La preocupación por la innovación confluyó con el interés creciente por la medición y el seguimiento del desarrollo tecnológico en la industria. Según García (2000): "Ese reconocimiento del papel clave que tiene la tecnología, y en particular la innovación tecnológica en la competitividad, ha generado en los últimos años un cambio en la cultura empresarial, (...), que les permitan efectivamente construir y sostener ventajas competitivas" (p. 159).

\section{La RSE vs Tecnología de información y la Comunicación}

La creciente importancia de las prácticas responsables socialmente de las compañías ha dado lugar a una demanda informativa por diversos usuarios, con el ánimo de conocer el comportamiento ético de la empresa y en el cual se advierte sobre la exigencia de equilibrio entre los ejes económico, social y medioambiental; por el que no puede optimizarse uno de los ejes (el económico) a costa de un mayor costo social 0 medio ambiental (Moneva \& Llena, 1996).

El análisis de cada dimensión debe evidenciar la definición de líneas estratégicas específicas, para que cada organización se despliegue de acuerdo al contexto de la comunidad donde se desenvuelve y favorece (Giménez \& Román, 2001).

Definiendo cada una de las dimensiones que integran la RSE, para Diem y Searles (1986), se tiene que la dimensión social implica la responsabilidad compartida y subsidiaria de inversionistas, directivos, colaboradores y proveedores para el cuidado y fomento de la calidad de vida en el trabajo y el desarrollo integral y pleno de todos ellos.

La dimensión ecológica, involucra la responsabilidad total sobre las consecuencias ambientales de sus procesos, productos y subproductos; y, por lo tanto, la prevención -y en su caso remedio- de los daños que causen o pudieran causar. Conlleva a la realización de acciones específicas para contribuir a la preservación y mejora ecológica común, para el bien de la humanidad actual y futura (Cheng, Li, Li, Holm, \& Zhai, 2013).

La responsabilidad de la dimensión económica se centra a la generación y distribución del valor agregado entre colaboradores y accionistas; asimismo, implica la generación y distribución de bienes y servicios útiles y rentables para la comunidad; además de su aportación a la causa pública vía la contribución impositiva (Tsiakis, 2009).

Ahora, según Asif, Searcy, Zutshi y Fisscher (2013) y Cruz (2009), las organizaciones están aprendiendo a producir y a consumir con valores que tienen que ver más con la responsabilidad social empresarial, las cuales generan ideas, ganas de innovar y de aplicarlas para salir de la crisis.

Las TIC, para Friedman (2009) son un facilitador eficaz para todo tipo de estrategias de negocio, por lo que no es ninguna sorpresa de que es útil para la implementación de la iniciativa de responsabilidad social empresarial de una organización. En la actualidad, las empresas tanto del sector público como del privado priorizan las TIC, donde se incluye la seguridad de la información, que es catalogada como uno de los bienes más preciados para la continuidad del negocio y el punto de diferencia con la competencia (Guziana, 2011) (Melchor, Lavín , \& Pedraza , 2012) (Díaz-Ricardo, Pérez-del Cerro, \& Proenza-Pupo, 2014).

En la actualidad, según Jackson y Culbertson (2013), Mayle (2009) y Monsalve, Aponte y Chaves (2014), la determinación del nivel de inseguridad (visto desde la óptica de vulnerabilidad y riesgo) de la información trasciende los niveles de su uso u operatividad, de forma que es necesario interpretar sus unidades de portabilidad y los medios por los que se transmite. Los motivos de implantar un Sistema de Gestión de Seguridad de la Información (SGSI) podrán estar derivados de aspectos regulatorios, legales, su estado, el tamaño de la firma, su ubicación geográfica, el tipo de negocio en que están inmiscuidas o el servicio que ofrecen. No puede implantarse de manera aislada, la organización debe considerar los riesgos 
de la organización y las estrategias globales de la firma. Siempre se debe tener en cuenta que el SGSI es una herramienta de la alta gerencia (Alexander, 2007) (Pereiro, 2011) (Viloria \& Blanco, 2009).

En ese sentido, como base según (Vera et al. , 2011), se tomara para el desarrollo de próximos trabajos, el impacto social positivo o negativo, que han generado las tecnologías de la información y la comunicación, como parte del desarrollo local y sustentable de las organizaciones.

\section{Conclusión}

Los procesos de innovación relacionados con la capacidad empresarial son los principales agentes del cambio tecnológico, por tal razón implican para las organizaciones una diversidad de actividades específicas y de prácticas gerenciales y administrativas, que no sólo se relacione con las áreas funcionales, sino que también involucre a potenciales consultores, proveedores, clientes, mercados e instituciones, entre otros; logrando acceder a procesos y prácticas de gestión del conocimiento que promuevan la generación de valor y el establecimiento de ventajas competitivas en los negocios.

De acuerdo a los resultados obtenidos a partir del análisis de los artículos referenciados sobre la RSE y las tres dimensiones que comprenden el desarrollo sustentable, las organizaciones con un comportamiento socialmente responsable ha rendido importantes ganancias. Ahora al integrarse en la sociedad, respetar el medio ambiente y ser socialmente responsable con los empleados y proveedores de la empresa, ha logrado dar a la misma, una imagen atrayente y diferenciada en el mercado donde compiten y se desenvuelven, convirtiéndose en una verdadera ventaja competitiva.

Las TIC son muy importantes en el contexto en la que se desarrolla la organización aplicadas en actividades de la comunidad. Así, todos los agentes implicados en la necesidad de conocer el alcance de la responsabilidad social derivada de la actividad empresarial toman conciencia, como un elemento para la toma de decisiones.

\section{Referencias Bibliográficas}

Albornoz, M. (2001). Política científica y tecnológica una visión desde América Latina. Revista Iberoamericana de Ciencia, Tecnología, Sociedad e Innovación(1).

Alderete, M. (2012). Medición de las tecnologías de la información y la comunicación en empresas de servicios de Colombia. Cuadernos de Administración, 25(45), 39-62.

Alderete, M. V., Jones, C., \& Morero, H. A. (2014). Factores explicativos de la adopción de las TIC en las tramas productivas automotriz y siderúrgica de Argentina. Pensamiento y Gestión (37), 1-40.

Alexander, A. (2007). Diseño y gestión de un sistema de seguridad de información (Vol. 1). Bogotá, Cundinamarca, Colombia: Alfaomega.

Asif, M., Searcy, C., Zutshi, A., \& Fisscher, 0. (2013). An integrated management systems approach to corporate social responsibility. Journal of Cleaner Production, 56, 7-17. doi:http://dx.doi.org/10.1016/j.jclepro.2011.10.034.

Bell, M., \& Pavitt, K. (1995). The development of technological capabilities, en I. ul Haque (comp.), Trade, Technology and International Competitiveness (1 ed.). Washington, D.C.: Banco Mundial.

Benavente H, J. M. (2005). Innovación tecnológica en Chile: dónde estamos y qué se puede hacer. Economía Chilena, $8(1)$.

Berné, C., García, M., Esperanza, U., \& Múgica, J. (2011). La influencia de las tic en la estructura del sistema de distribución turístico. Cuadernos de Turismo (28), 9-22.

Cano, J. (2013). Inseguridad de la información: Una visión estratégica. Bogota, Cundinamarca, Colombia: Alfaomega.

Cheng, L., Li, Y., Li, W., Holm, E., \& Zhai, Q. (2013). Understanding the violation of IS security policy in organizations: An integrated model based on social control and deterrence theory. Computers \& Security, 39, 447-459. doi:http://dx.doi.org/10.1016/j.cose.2013.09.009

Correa, A. A., Gómez, R. A., \& Cano, J. A. (2010). Gestión de almacenes y tecnologias de la información y la comunicación. Estudios Gerenciales, 26 (117), 145-171.

Cruz, J. (2009). The impact of corporate social responsibility in supply chain management: Multicriteria decision-making approach. Decision Support Systems, 48 (1), 224-236. doi:http://dx.doi.org/10.1016/j.dss.2009.07.013

Dagnino, R., \& Hernán, T. (1999). La política científica y tecnológica en América Latina. Dagnino, Renato y Thomas, Hernán; La política cient redes, 6 (13). 
Responsabilidad social empresarial e innovación: Una mirada desde las tecnologías de la información y comunicación en organizaciones

Díaz-Ricardo, Y., Pérez-del Cerro, Y., \& Proenza-Pupo, D. (2014). Sistema para la Gestión de la Información de Seguridad Informática en la Universidad de Ciencias Médicas de Holguín. Ciencias Holguín, 20 (2), 1-14. Recuperado el 10 de 02 de 2015

Diem, R., \& Searles, J. (1986). Social responsibility in technological environments. Comput. E\&C, 10 (1), 215-219. doi:http://dx.doi.org/10.1016/0360-1315(86)90075-8

Dosi, G. (1988). Sources, procedures and microeconomic effects of innovation. Journal of Economic Literature, 26 (3).

Ezequiel, E. (2014). Capacidades Tecnológicas: La piedra angular para la competitividad y la rentabilidad empresarial. Revista de Economía y Comercio Internacional (4), 17-21.

Freeman, C. (1994). The economics of technical change. Cambridge Journal of Economics, 18 (5), 463-514.

Friedman, H. (2009). The role of information technology in corporate social responsibility. Proceedings For The Northeast Region Decision Sciences Institute (NEDSI), 234-239. Recuperado el 10 de 02 de 2015

García Muiña, F. E., \& Navas Lopéz, J. E. (2007). Las capacidades Tecnologiccas y los resultados empresariales. Un estudio empririco del sector biotecnologico español. Cuadernos de Economia y Direccion de la empresa (32), 177-210.

García, H. T. (2000). GEstión tecnológica y desarrollo tecnológico. (U. d. Antioquia, Ed.) Revista Facultad de Ingeniería (21), 158-177.

Gargallo, A., \& Pérez , J. (2009). El papel de las tecnologías de la información y la comunicación en las empresas de economía social. Revesco, 90-116.

Giménez, E., \& Román, A. (2001). Vigilancia tecnológica e inteligencia competitiva: conceptos, profesionales, servicios y fuentes de información. El profesional de la información, 10 (5), 11-20. Recuperado el 10 de 02 de 2015.

González , L., \& Casalet, M. (2004). Las tecnologías de la información en las pequeñas y medianas empresas mexicanas. Scripta Nova, 8 (170 (21)).

Guziana, B. (2011). Is the Swedish environmental technology sector 'green'? Journal of Cleaner Production, 19 (8), 827835. doi:http://dx.doi.org/10.1016/j.jclepro.2010.09.007

Ibarra, L., Casas, E., \& Olivas , E. (2012). La Responsabilidad Social Empresarial: Una estrategia enfocada al desarrollo sustentable. Caso: Las ESR de Hermosillo, Sonora. Revista Iberoamericana de Contaduría, Economía y Administración, 1 (1). Recuperado el 30 de 01 de 2015.
Jackson, A., \& Culbertson, S. (2013). Bad employees or bad policies: what can organizations do to stop misuse of information technology resources? Academy of Management Perspectives, 27 (1). doi:http://dx.doi.org/10.5465/ amp.2013.0026

Lugones, G. E., Gutti, P., \& Le Clech, N. (2007). http:// repositorio.cepal.org/. Recuperado el 15 de Enero de 2015, de http://repositorio.cepal.org/bitstream/ handle/11362/5014/S0700876_es.pdf?sequence=1

Mayle, D. (2009). Change management (Vol. 4). Sage Publications. Recuperado el 10 de 02 de 2015.

Melchor , J., Lavín , J., \& Pedraza , N. (2012). Seguridad en la administración y calidad de los datos de un sistema de información contable en el desempeño organizacional. Contaduría y Administración, 57 (4), 11-34. Recuperado el 10 de 02 de 2015.

Milesi, D., Moori, V., Robert, V., \& Yoguel, G. (2007). Desarrollo de ventajas competitivas: pymes exportadoras exitosas en Argentina, Chile y Colombia. (N. CEPAL., Ed.) Revista de la CEPAL 92 (92), 25 - 43.

Moneva, J. M., \& Llena, F. (1996). Analisis de la informacion sobre responsabilidad social en las empresas industriales que cotizan en bolsa. Revista Esparola de Rnanciacion y Contabilidad, 25 (87), 361-401. Recuperado el 30 de 01 de 2015.

Monsalve, J., Aponte, F., \& Chaves, D. (2014). Estudio y gestión de vulnerabilidades informáticas para una empresa privada en el departamento de Boyacá (Colombia). Revista Facultad de Ingeniería, 23 (37), 65-72. Recuperado el 10 de 02 de 2015.

Montaño, J., \& Montoya, J. (2010). Utilización del comercio electrónico en las medianas empresas de Palmira - Valle del Cauca - Colombia. Entramado, 6 (2), 56-73.

Nelson, R. (1991). Why do firms differ, and how does it matter? Strategic Management Journal, 12 (1).

Parada , J. (2001.). Programa especial de ciencia y tecnología 2001-2006. Consejo Nacional de Ciencia y Tecnología, CONACYT, Mexico. Recuperado el 22 de enero de 2015, de http://www.cepal.org/iyd/noticias/pais/8/31458/ Mexico_Doc_4.pdf

Pereiro, M. (2011). Seguridad de la información en el Uruguay: políticas de Estado en la administración pública. Revista de la Asociación de Escribanos del Uruguay, 97, 137-156. Recuperado el 01 de 02 de 2015. 
Pérez, M. V., \& Rodríguez, F. M. (2004). L0s avances en la medición del desarrollo tecnológico en la industria colombiana. Revista CTS, 1 (2), 137-136.

Pietrobelli, C. (1996). A note on the theory of technological capabilities and developing countries, dynamic comparative advantage in manufactures. Roma: Università di Roma "Tor Vergata", inédito.

Poriet, Y., \& Martínez, 0. (2014). Competencias tecnológicas en la gerencia universitaria. Orbis, 9(27), 47-67.

Saavedra, M., \& Tapia, B. (2013). El uso de las tecnologías de información y comunicación TIC en las micro, pequeñas y medianas empresas (MIPyME) industriales mexicanas. Enl@ce: Revista Venezolana de Información, Tecnología y Conocimiento, 10 (1).

Salazar, D., Vanegas, M., \& Arboleda, D. (2009). Nuevas tecnologías en la comunicación interna en empresas del Valle de Aburrá. Revista Lasallista de Investigación, 6 (1).

Sánchez , A. (2011). Manual de redacción académica e investigativa: cómo escribir, evaluar y publicar artículos. Medellin, Antioquia, Colombia: Fundación Universitaria Católica del Norte.

Sandulli, F., Fernández, J., López, J., \& Rodríguez, A. (2007). El impacto del uso efectivo de las TIC sobre la eficiencia técnica de las empresas españolas. Estudios Gerenciales, 23 (103), 65-84.
Scheel Mayenberger, C., \& Rivera Gonzales, A. E. (2009). Utilizacion de las TIC y su impacto en la competitividad de las empresas latinoamericanas. Univ. Empresa, 8 (16), 71-93.

Treviño, L. C. (2002.). Innovación y competitividad empresarial. Aportes: Revista de la Facultad de Economía-Buap. (20), $55-65$.

Tsiakis, T. (2009). Contribution of corporate social responsibility to information security management. Information Security Technical Report, 14 (4), 217-222. doi:http:// dx.doi.org/10.1016/j.istr.2010.05.001

Venteño, M., De la Rosa, M., Casas, F., Trujillo, J., \& Fuentes, V. (2010). El acceso y uso de las tecnologías de información y comunicación por la empresa: el caso de la banca en México. Biblioteca Universitaria, 13 (1), 36-54.

Vera, P., Labarcés, C., \& Chacón, E. (2011). Influencia de las TIC en las organizaciones: Cambios y aparición de nuevas formas organizativas. Clío América, 5 (10), 228-244.

Viloria, 0., \& Blanco, W. (2009). Modelo sistémico de la seguridad de la información en LAS. Revista Venezolana de Análisis de Coyuntura, 15 (1), 219-240. 